\title{
Predictive modelling of impurity seeded plasmas in JET
}

\author{
M Z Tokar ${ }^{1}$, H Nordman ${ }^{2}$, J Weiland ${ }^{2}, J_{\text {Ongena }}^{3}$, V Parail ${ }^{4}$, \\ B Unterberg ${ }^{1}$ and contributors to the EFDA-JET Workprogramme \\ ${ }^{1}$ Institut fur Plasmaphysik, Association EURATOM-FZJ, Julich 52425, Germany \\ 2 Chalmers University of Technology, Association EURATOM-NFR, Göteborg, Sweden \\ ${ }^{3}$ ERM-KMS, Association 'EURATOM-Belgian State', Brussels, Belgium \\ ${ }^{4}$ EURATOM/UKAEA Fusion Association, Culham Science Centre, Abingdon, UK
}

Received 16 January 2002

Published 27 August 2002

Online at stacks.iop.org/PPCF/44/1903

\begin{abstract}
Seeding of impurities into JET resulted in a pronounced modification of plasma confinement properties. In order to analyse the underlying mechanisms a predictive transport modelling has been performed by means of two approaches. (i) The Chalmers advanced fluid model for temperature gradient driven instabilities is applied to determine all transport coefficients necessary for transport modelling. In the present code computations the radial profiles of the particle densities and temperatures are computed using experimentally measured sources. The computations give good agreement of the temperature profiles with the measured ones. (ii) The Jülich transport model, used earlier to simulate the plasma evolution in TEXTOR radiative improved mode, has been amended by a proper description of trapped electron transport, which is important at low collisionality typical for JET. This model incorporated into the transport code RITM, which self-consistently computes particle sources due to plasma recycling and impurity ionization, reproduces well the peaking in density and pressure profiles caused by impurity seeding.
\end{abstract}

\section{Introduction}

The possibility to improve confinement in tokamaks by seeding of impurities has been successfully realized in several devices [1-3]. Earlier trials on JET [4] were not, however, encouraging and sowed doubts that this operation mode can be achieved in large machines. New series of experiments performed under the EFDA agreement have shown that also in JET a positive effect of impurity seeding can be attained through an optimization of the puffing scenario [5-7]. For example, carefully dozed puffs of argon made it possible to maintain good confinement in the H-mode discharges at an electron density close to the Greenwald limit. Another type of discharge, in which neon impurity was seeded, is the L-mode. This scenario 
(a)

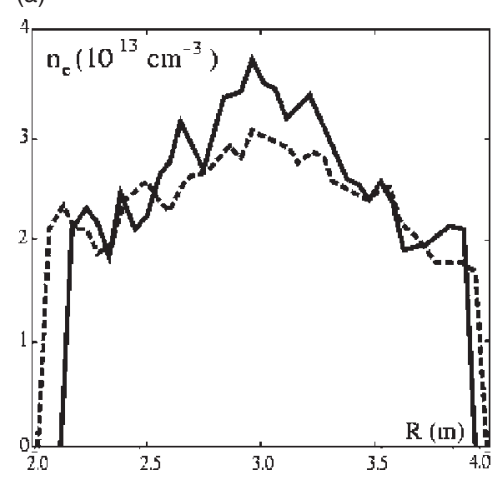

(b)

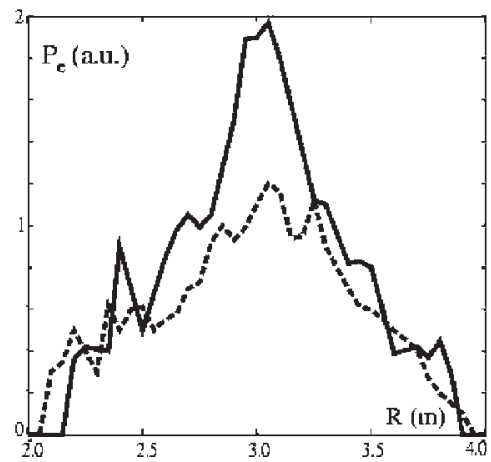

Figure 1. Electron density $(a)$ and pressure $(b)$ profiles in JET shot 50329 before (- - - -) and after $(-)$ neon puff.

was elaborated on TEXTOR [2] and DIII-D [3], where the stage of improved confinement, the so-called radiative improved (RI) mode, was achieved starting from the L-mode conditions. In this case the most characteristic features of the RI-mode such as peaking of density and pressure have been seen in JET [5-7]. As an example, figure 1 shows the radial profiles of plasma parameters measured in JET shot 50329 before and after neon puff.

In this contribution, the results of theoretical analysis and numerical modelling both for Land H-mode conditions are presented; however, the former ones are treated more intensively. This is because the theoretical models applied for our study were developed mainly to analyse the RI-mode in TEXTOR and up to now they do not include the edge barrier. Therefore, in the case of $\mathrm{H}$-mode conditions only the internal plasma region was modelled.

\section{Predictive transport modelling}

\subsection{Transport codes}

For predictive transport modelling of JET plasmas seeded with impurities, two transport codes, RITM [8] and Chalmers model [9, 10], have been applied. Both codes solve continuity equations for electrons,

$$
\frac{\partial n_{\mathrm{e}}}{\partial t}+\frac{1}{r g_{1}} \frac{\partial}{\partial r}\left(r g_{2} \Gamma_{\perp}^{\mathrm{e}}\right)=S_{\mathrm{n}}+\sum_{Z} Z S_{Z},
$$

and impurity ions,

$$
\frac{\partial n_{z}}{\partial t}+\frac{1}{r g_{1}} \frac{\partial}{\partial r}\left(r g_{2} \Gamma_{\perp}^{Z}\right)=S_{Z}
$$

with $r$ being the minor radius of the magnetic surface, $n_{\mathrm{e}, Z}$, and $\Gamma_{\perp}^{\mathrm{e}, Z}$ the densities of electrons and impurity species of the charge $Z$ and their fluxes perpendicular to the magnetic surfaces; $S_{\mathrm{n}}$ is the net electron source due to ionization of hydrogen neutrals and $S_{Z}$ is the source of the impurity species. The density and flux of the background ions are computed from the quasi-neutrality conditions:

$$
n_{\mathrm{i}}=n_{\mathrm{e}}-\sum Z n_{Z}, \quad \Gamma_{\perp}^{\mathrm{i}}=\Gamma_{\perp}^{\mathrm{e}}-\sum Z \Gamma_{\perp}^{Z}
$$

In the code RITM, the deuteron source is calculated self-consistently from the velocity distribution function $f_{j}\left(v_{r}\right)$ of neutral particles produced by recycling processes and neutral 
beams. This is determined by the kinetic equation

$$
\frac{1}{g_{1}} \frac{\partial}{\partial r}\left(v_{r} f_{j}\right)=S_{j}-v_{j} f_{j}
$$

where $j=1-4$ corresponds to neutral molecules and reflected atoms, which are puffed or generated at divertor plates and penetrate through the separatrix, Franck-Condon and hot atoms arising in the plasma in processes of ionization, dissociation, charge-exchange; $S_{j}$ and $v_{j}$ are sources and frequencies of disintegrative processes for corresponding kinds of neutrals. Equation (2) are solved for all charge states of $\mathrm{He}, \mathrm{C}, \mathrm{O}, \mathrm{Ne}$ and $\mathrm{Si}$ impurities; impurity neutrals, puffed and recycling in the case of $\mathrm{Ne}$ or eroded from the wall in the case of intrinsic impurities, are described in diffusion approximation. In the Chalmers model $S_{\mathrm{n}}$ is taken from TRANSP modelling and only one effective impurity species is included.

The heat transport is governed by equations for the electron and ion temperatures $T_{\mathrm{e}}$ and $T_{\mathrm{i}}$ and the latter is assumed the same for all ion species:

$$
\begin{aligned}
& \frac{3}{2} \frac{\partial n_{\mathrm{e}} T_{\mathrm{e}}}{\partial t}+\frac{1}{r g_{1}} \frac{\partial}{\partial r}\left[r g_{2}\left(1.5 \Gamma_{\perp}^{\mathrm{e}} T_{\mathrm{e}}-\kappa_{\perp}^{\mathrm{e}} \frac{\partial T_{\mathrm{e}}}{\partial r}\right)\right]=\frac{J^{2}}{\sigma}+Q_{\mathrm{au}}^{\mathrm{e}}-Q_{\mathrm{ei}}-Q_{\mathrm{en}}-Q_{\mathrm{eI}}, \\
& \frac{3}{2} \frac{\partial n_{\Sigma} T_{\mathrm{i}}}{\partial t}+\frac{1}{r g_{1}} \frac{\partial}{\partial r}\left[r g_{2}\left(1.5 \Gamma_{\perp}^{\Sigma} T_{\mathrm{i}}-\kappa_{\perp}^{\Sigma} \frac{\partial T_{\mathrm{i}}}{\partial r}\right)\right]=Q_{\mathrm{au}}^{\mathrm{i}}+Q_{\mathrm{ei}}+Q_{\mathrm{in}}
\end{aligned}
$$

where $n_{\Sigma}=n_{\mathrm{i}}+\Sigma n_{Z}, \Gamma_{\Sigma}=\Gamma_{\mathrm{i}}+\Sigma \Gamma_{Z}, Q_{\mathrm{au}}^{\mathrm{e}, \mathrm{i}}$ is the density of heat sources in electrons and ions due to additional heating from NBI and ICRH computed by TRANSP, $Q_{\text {ei }}, Q_{\text {en }}, Q_{\text {eI }}$ the energy losses from electrons due to coulomb collisions with ions, excitation and ionization of neutrals and impurities, respectively (in RITM the latter two terms are computed self-consistently, in the Chalmers model they are taken from measurements and TRANSP results), $Q_{\text {in }}$ the energy exchange between main ions and neutrals.

The time evolution of the current density profile including both Ohmic and bootstrap contributions is computed from current diffusion equation with the electro-conductivity calculated by taken neoclassical corrections into account. In order to determine the metric coefficients $g_{1,2}$, the shift of magnetic surface centre is computed in RITM from the GradShafranov equation in cylindrical approximation and the ellipticity and triangularity of surfaces are prescribed analytically. The Chalmers model is coupled to the code EFIT for the determination of these parameters from magnetic signals.

The boundary conditions for the transport equations at the separatrix imply in the code RITM given e-folding lengths for the densities and temperatures. In the Chalmers model the plasma temperatures and densities are assumed equal to the measured one at a certain magnetic surface at the plasma edge.

\subsection{Transport models}

2.2.1. RITM code. RITM operates with a half-empirical model for anomalous transport. This includes the contribution from ion temperature gradient (ITG) [9] and dissipate trapped electron (DTE) $[9,11]$ unstable modes which are mainly of importance in the plasma central region, $\rho=r / a \leqslant 0.9$; at the plasma edge the transport is due to drift resistive ballooning modes [12] and turbulence caused by electric currents in the scrape-off layer [13]. The latter is assumed decaying from the separatrix into the confined volume at a distance of the pressure decay length at the separatrix. With dependences on the ion effective charge and electron density gradient, the ITG-DTE model has allowed to simulate firmly the plasma transition into the RI-mode by neon seeding in TEXTOR [14-16]. For description of JET plasmas of a 
low collisionality, this model has been amended by a proper description of the DTE transport according to [11].

The particle fluxes contain both diffusive and convective parts:

$$
\Gamma_{\perp}^{\mathrm{e}, Z}=-D_{\perp}^{\mathrm{e}, Z} \frac{\partial n_{\mathrm{e}, Z}}{\partial r}+V_{\perp}^{\mathrm{e}, Z} n_{\mathrm{e}, Z}
$$

the electron diffusivity includes contributions from ITG, DTE and edge turbulence:

$$
D_{\perp}^{\mathrm{e}}=D_{\perp}^{\mathrm{ITG}} f_{\mathrm{tr}}+D_{\perp}^{\mathrm{DTE}}+D_{\perp}^{\text {edge }}
$$

here $f_{\text {tr }}$ is the fraction of trapped electrons computed by taking into account both inhomogeneity of the magnetic field and detrapping by coulomb collisions; this factor reflects that ITG turbulence leads to particle transport mainly through stochastization of banana orbits [17]. The electron pinch-velocity is assumed to be governed by the magnetic shear [18]:

$$
V_{\perp}^{\mathrm{e}}=\left(D_{\perp}^{\mathrm{ITG}} f_{\mathrm{tr}} \frac{4 r}{3 R}+D_{\perp}^{\mathrm{DTE}}\right) \frac{\mathrm{d} \ln q}{\mathrm{~d} r} ;
$$

the diffusivity of impurities is adopted the same as for the main particles and their pinch velocity is neoclassical: $D_{\perp}^{Z}=D_{\perp}^{\mathrm{e}}, V_{\perp}^{Z}=V_{\perp}^{Z \text {,neo }}$; the transport of main ions is not prescribed and their flux is computed from the ambipolarity condition $(3 b)$. The anomalous transport coefficients are computed in mixing length approximation,

$$
D_{\perp}^{\mathrm{ITG}, \mathrm{DTE}}=\frac{\gamma_{\max }}{k_{\perp, \max }^{2}} \times q^{2}
$$

$[9,11]$, with the growth rates $\gamma_{\max }$ corresponding to the most unstable modes. The used dependence of the growth rates on plasma parameters, their gradients, etc, can be found in [16].

In the present RITM simulations the effect of the radial electric field on the growth rate of drift instabilities was not taken into account, because there is not any model in the present code version for the ion velocities on magnetic surfaces, which are needed for determination of $E_{\mathrm{r}}$. The analysis by the code for gyro-kinetic stability (GKS) performed for impurity seeded discharges in DIII-D and JET [7, 19] indicates that this effect can be an essential ingredient of the L-RI bifurcation mechanism. Earlier this idea was put forward to explain the L-RI transition in discharges with unbalanced NBI injection when toroidal rotation provides a large contribution to the reduction in growth rate, $\Omega_{E \times B}$ [20]. However, GKS results also show that namely direct effect of impurities on the growth rate of instabilities gives an initial 'kick' to the reduction of transport $[7,19]$. The present modelling allows one to judge how effective is this 'kick' for the plasma evolution without consequent support from the radial electric field. Recent computations with RITM for RI-plasmas in TEXTOR [14] showed that it alone could be enough for a strong suppression of ITG transport. This is in agreement with observations of RI-mode of a good quality in TEXTOR discharges with balanced NBI [21] when a large enough $E_{\mathrm{r}}$ does not exist and only direct effect of impurities can be responsible for the L-RI transition. For discharges with strong toroidal rotation in TEXTOR, a modelling with a reduced set of transport equations has shown [14] that including the radial electric field results in a better agreement with experiment, in particular, concerning the speediness of the L-RI transition and the width of the plasma region where anomalous transport is reduced. For JET such an analysis will be done in near future when a self-consistent model for the radial electric field and toroidal and poloidal plasma velocities [22] will be introduced into the present RITM version.

2.2.2. Chalmers. This transport code is based on the advanced fluid model $[9,10]$ for temperature gradient driven instabilities, which allows to compute all anomalous transport coefficients necessary for transport modelling. This code was successfully run before to 
simulate standard L- and H-mode discharges in JET and ASDEX-U. The version used in the present simulation includes the stabilizing influence of impurities and sheared plasma rotation and describes transport due to ITG modes, collisionless trapped electron modes and impurity modes. Artificial diffusivity is used for $r / a<0.3$ to model inner core transport since the contribution from the drift waves is usually small in this region (due to flat profiles). However, the results in the region $r / a>0.3$ are rather insensitive to this choice [10].

\section{Results of transport modelling}

\subsection{RITM}

The results of computations with RITM presented in figure 2 reproduce the peaking of the density and pressure profiles, which follows the neon seeding into the L-mode discharge 50329 in JET. The former is caused by reduction in the electron diffusivity, which is also demonstrated in figure 2. This reduction is mostly due to decrease of $D_{\perp}^{\mathrm{ITG}}$ with increasing $Z_{\text {eff }}$ (see [14-16]). The modification of the components of the electron flux density and the ratio $V_{\perp}^{\mathrm{e}} / D_{\perp}^{\mathrm{e}}$ are demonstrated in figure 3 . The significant increase in the latter in the range $r=70-80 \mathrm{~cm}$ results in a more peaked density profile. In computation the process of peaking and narrowing of the plasma region dominated by ITG turbulence continues also for later times; however, in experiment it is terminated by expelling of impurities by sawtooth activity. The separation of the plasma radius into two zones, where ITG and DTE transport dominate, are in agreement with GKS analysis performed for JET neon seeded shot 53156 [7].

The results of modelling allow to interpret the necessity of a significantly higher plasma effective charge in JET, 5-7, than in DIII-D and TEXTOR, 2-3, in order to get an RI-mode effect starting from L-mode conditions. The ratio $V_{\perp}^{\mathrm{e}} / D_{\perp}^{\mathrm{e}}$, which controls the density peaking, is governed in the RITM model by the value $D_{\perp}^{\mathrm{DTE}} / D_{\perp}^{\mathrm{ITG}}$ and for a transition to the RI-mode the latter should exceed a critical level [14-16]. On the one hand for plasmas with low collision collisionality as in JET, where the electron drift frequency exceeds the effective collision frequency of trapped particles, $D_{\perp}^{\mathrm{DTE}} \propto n_{\mathrm{e}}$ [11]. On the other hand $D_{\perp}^{\mathrm{ITG}}$ decreases with increasing $Z_{\text {eff }}$ and ITG transport is suppressed completely when the plasma effective charge exceeds a certain critical level [15]. Thus the lower the plasma density, the higher the $Z_{\text {eff }}$, at which $D_{\perp}^{\mathrm{DTE}} / D_{\perp}^{\mathrm{ITG}}$ approaches its critical level. Since the plasma density in JET L-mode is

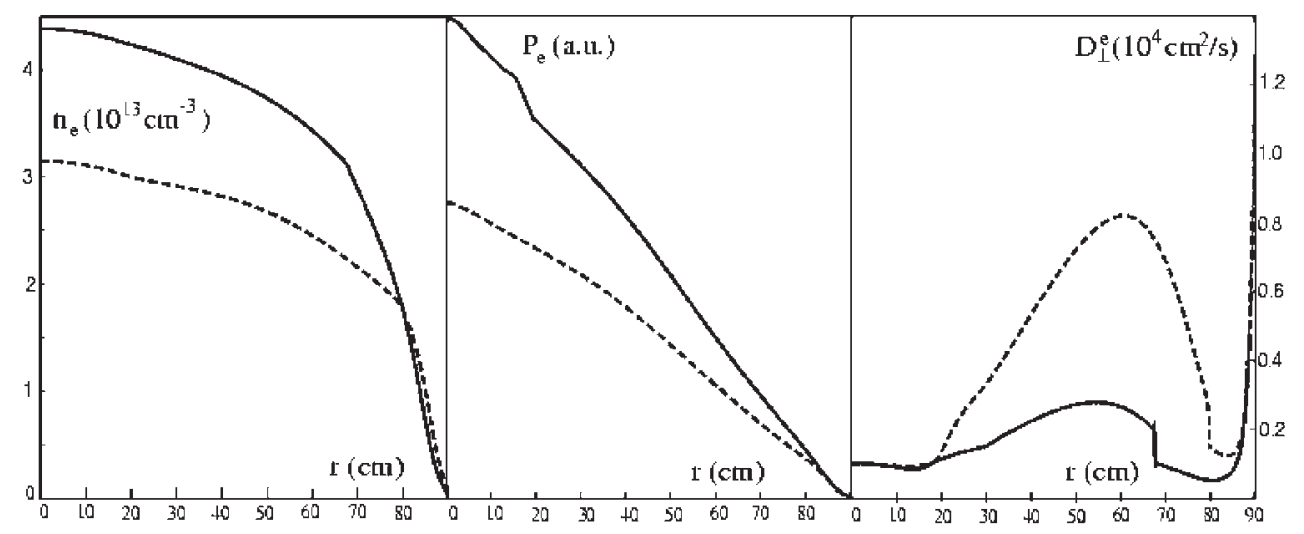

Figure 2. Radial profiles of plasma density, pressure and diffusivity computed by RITM for JET shot 50329 before (- - - ) and after (_- ) neon puff. 


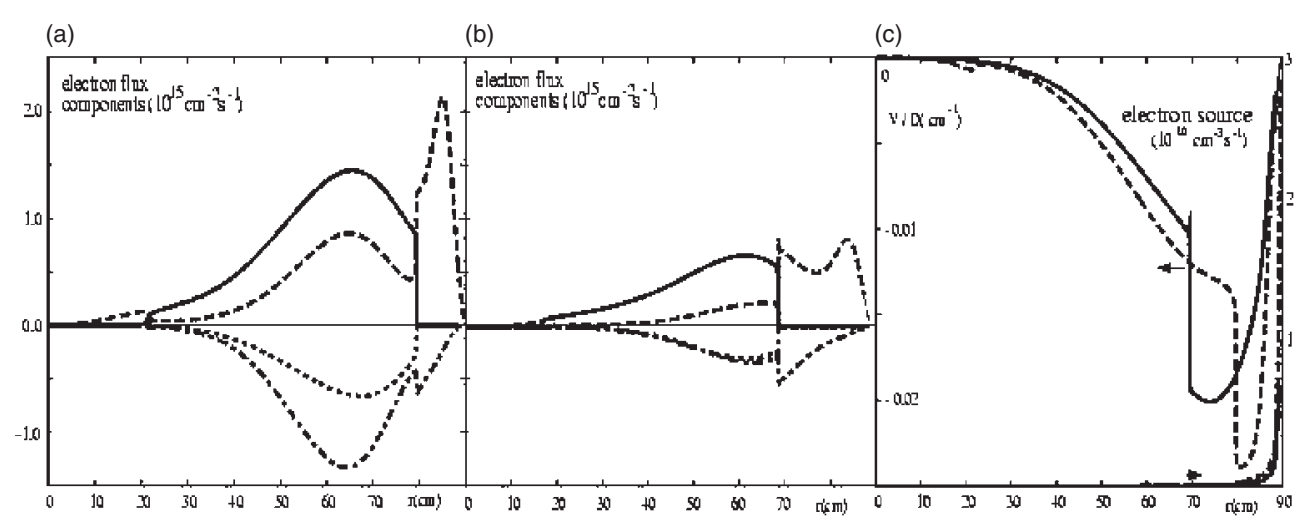

Figure 3. Computed profiles of the electron flux density components: ITG and DTE diffusion ( $\longrightarrow$ and - - - ) and convection (pointed and point-and-dashed curves) in L-mode $(a)$ and after neon puff $(b)$ and $V / D$ ratio with electron source density $(c)$.

significantly less than in DIII-D and TEXTOR, the required $Z_{\text {eff }}$ is larger in JET.

In concluding notice that the particle source due to recycling and ionization of impurities, which is also shown in figure $3(c)$, is localized at the very edge and even is decreased in the RI-stage. Therefore, its effect on the process of density peaking is unessential. The source from NBI is too weak in JET to cause peaking. Moreover, experiments with slow density peaking under conditions of moderate gas puffing [23] show that peaking increases with reducing NBI power and decreasing central particle source.

\subsection{Chalmers model}

Figures $4(a)$ and $(b)$ show the radial profiles of the predicted ion temperature, electron temperature and density are compared with experimental data at $t=46.1 \mathrm{~s}$ in L-mode shot \#50329, after the neon puff. The temperature profiles are reasonably well reproduced by the simulation with time-averaged root-mean-square errors of $\sigma\left(T_{\mathrm{i}}\right)=15.6 \%, \sigma\left(T_{\mathrm{e}}\right)=10.2 \%$ and $\sigma\left(n_{\mathrm{e}}\right)=6.4 \%$. However, the electron density peaking observed inside $r / a=0.3$ is not well reproduced. In this region, the code uses a fixed artificial diffusivity $D_{\text {art }}=1 \mathrm{~m}^{2} \mathrm{~s}^{-1}$ throughout the simulation, to model inner core transport. A reduction in transport is observed in the simulations after the neon puff, which is followed by an increase of the effective charge, $Z_{\text {eff }}$, in the plasma edge. In addition, the electron density is reduced at the edge. This reduction in $n_{\mathrm{e}}$ enters the simulation dynamically through the boundary value and leads to a reduction of $\eta_{\mathrm{i}}=L_{\mathrm{n}} / L_{T_{\mathrm{i}}}$. The result is a reduction of the ITG driven flow throughout the plasma cross-section. These results are in qualitative agreement with those obtained by the code RITM. Figure 4(c) presents the results for shot 52136 when argon was puffed into H-mode plasma. In this case the simulations reproduce well the relatively flat density profile in the plasma core.

Computations for the L-mode shot 50329 were performed both by neglecting and taking into account the reduction of the linear growth rates by the $E \times B$ shearing. The latter was calculated from the measured toroidal velocity and neoclassical prediction for the poloidal ion velocity. The results indicate that the stabilizing effects of sheared plasma rotation are subdominant in the present simulation, which agrees with a previous study for TEXTOR $[14,15]$. This result is not in line with the conclusion drawn from GKS modelling [7] for measured plasma parameter profiles on the dominant role of the radial electric field in the 


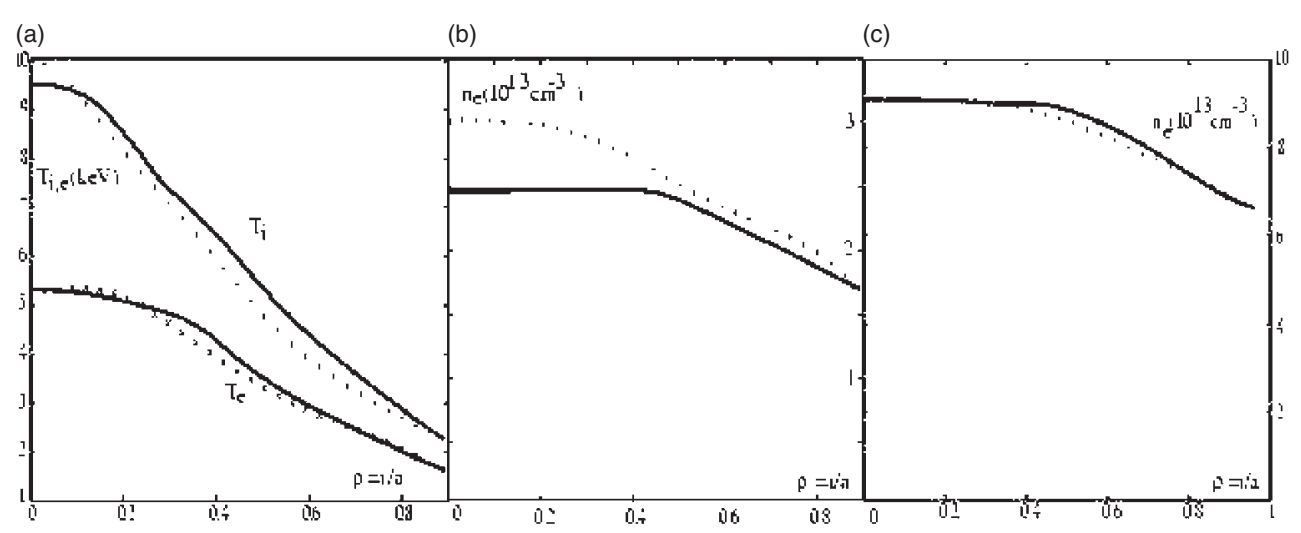

Figure 4. Comparison of computed (with the Chalmers) model (solid lines) and measured (crosses and pluses) profiles of charged particle temperatures $(a)$ and density $(b)$ in shot $\# 50329$ at $t=46.1 \mathrm{~s}$ after neon puff into L-mode and in shot \#52132 after argon puff into H-mode plasma (c).

confinement improvement by impurity seeding into JET.

Although the main features of the transport seem to be reproduced by the present simulation with the Chalmers model, the results indicate that a more detailed model for inner core particle transport and a more realistic treatment of the impurity dynamics, including the self-consistent evolution of more than one impurity species, are needed in order to draw more detailed conclusions from simulations of impurity seeding into JET.

\section{Conclusion}

Predictive modelling with the code RITM by taking into account only the direct effect of impurities on the anomalous transport through increase in the ion effective charge, reproduces well the peaking of density and pressure profiles by seeding of neon into JET L-mode plasma. The necessity to have a higher effective plasma ion charge in JET than in DIII-D and TEXTOR to trigger the L-RI transition is interpreted by a significantly lower plasma density: low collisionality results in a very weak pinch-velocity due to DTE modes and a stronger reduction of the ITG diffusion by increased $Z_{\text {eff }}$ is required to activate density peaking.

The Chalmers model reproduces well the ion and electron temperature profiles after neon puffing into the L-mode discharge in JET; however, density peaking was not traced in the plasma core. Computation with and without radial electric field shows that this plays subdominant role in the evolution of impurity seeded plasmas and the direct effect of impurities is more important. This is in agreement with RITM results but in contradiction to the results of GKS analysis. For the case of argon puffing into H-mode, when the density profile remains relatively flat, the Chalmers model results in a good agreement with measured density profile.

For future development a model for the radial electric field should be incorporated into the code RITM to take into account its effect on the growth rate of drift instabilities. This is especially of importance for discharges with unbalanced NBI where plasma toroidal rotation contributes strongly to $E_{\mathrm{r}}$. Such an amendment is necessary in order to finally clarify the question which channel of turbulence suppression, direct effect of impurities or radial electric field, is most important for the transition to RI plasmas. 


\section{References}

[1] Lazarus E A et al 1984 J. Nucl. Mater. 12161

[2] Weynants R R et al 1999 Nucl. Fusion 391637 Ongena J et al 2001 Phys. Plasmas 82188

[3] McKee G R et al 2000 Phys. Plasmas 71870

[4] Matthews G F et al 1999 Nucl. Fusion 3919

[5] Maddison G P et al Radiating-edge plasma experiments on JET Nucl. Fusion submitted

[6] Ongena J et al 2001 Plasma Phys. Control. Fusion 43 A11

[7] Jackson G L et al 2001 Eur. Conf. Abstracts A 25993

[8] Tokar M Z 1994 Plasma Phys. Control. Fusion 361819

[9] Weiland J 2000 Collective Modes in Inhomogeneous Plasmas. Kinetic and Advanced Fluid Theory (Bristol: Institute of Physics Publishing)

[10] Strand P et al 1998 Nucl. Fusion 28545

[11] Kadomtsev B B and Pogutse O P 1971 Nucl. Fusion 1167

[12] Gusdar P N et al 1993 Phys. Fluids B 53712

[13] Endler M, Giannone L, McCormick K, Niedermeyer H, Rudyj A, Thiemer G, Tsosis N and the ASDEX team 1995 J. Nucl. Mater. 220-222 293

[14] Tokar M Z, Jaspers R, Koslowski H R, Krämer-Flecken A, Messiaen A M, Ongena J, Rogister A A, Unterberg B and Weynants R R 1999 Plasma Phys. Control. Fusion 41 B317

[15] Tokar M Z, Ongena J, Unterberg B and Weynants R R 2000 Phys. Rev. Lett. 84895

[16] Kalupin D, Tokar M Z, Dumortier P, Messiaen A, Reiser D, Soldatov S, Unterberg B, van Wassenhove G and Weynants R R 2001 Plasma Phys. Control. Fusion 43945

[17] Horton W et al 1985 Plasma Phys. Control. Fusion 27937

[18] Baker D R and Rosenbluth M N 1998 Phys. Plasmas 52936

[19] Murakami M et al 2001 Nucl. Fusion 41317

[20] Tokar M Z, Jaspers R and Unterberg B 1998 Nucl. Fusion 38961

[21] Messiaen A M et al 1996 Nucl. Fusion 36825

[22] Claassen H A, Gerhauser H, Rogister A and Yarim C 2000 Phys. Plasmas 73699

[23] Valovic M et al 2001 Eur. Conf. Abstracts A 25957 